\title{
Macrophage Activation Syndrome in Patients Affected by Adult-onset Still Disease: Analysis of Survival Rates and Predictive Factors in the Gruppo Italiano di Ricerca in Reumatologia Clinica e Sperimentale Cohort
}

\author{
Piero Ruscitti, Daniela Iacono, Francesco Ciccia, Giacomo Emmi, Paola Cipriani, \\ Rosa Daniela Grembiale, Federico Perosa, Lorenzo Emmi, Giovanni Triolo, \\ Roberto Giacomelli, and Gabriele Valentini
}

\begin{abstract}
Objective. Macrophage activation syndrome (MAS) is a reactive form of hemophagocytic lymphohistiocytosis, which can complicate adult-onset Still disease (AOSD). We investigated AOSD clinical features at the time of diagnosis, to assess predictors of MAS occurrence. Further, we analyzed the outcomes of patients with AOSD who experience MAS.

Methods. Patients with AOSD admitted to any Gruppo Italiano di Ricerca in Reumatologia Clinica e Sperimentale center were retrospectively analyzed for features typical of AOSD, MAS occurrence, and their survival rate.

Results. Of 119 patients with AOSD, 17 experienced MAS (12 at admission and 5 during followup). Twelve patients with MAS at first admission differed from the remaining 107 in prevalence of lymphadenopathy and liver involvement at the time of diagnosis. In addition, serum ferritin levels and systemic score values were significantly higher in the patients presenting with MAS. At the time of diagnosis, the 5 patients who developed MAS differed from the remaining 102 in the prevalence of abdominal pain, and they showed increased systemic score values. In the multivariate analysis, lymphadenopathy (OR 7.22, 95\% CI 1.49-34.97, $\mathrm{p}=0.014)$ and abdominal pain $(\mathrm{OR} 4.36,95 \% \mathrm{CI}$ $1.24-15.39, \mathrm{p}=0.022)$ were predictive of MAS occurrence. Finally, MAS occurrence significantly reduced the survival rate of patients with AOSD $(\mathrm{p}<0.0001)$.

Conclusion. MAS occurrence significantly reduced the survival rate in patients with AOSD. Patients with MAS at baseline presented an increased prevalence of lymphadenopathy and liver involvement, as well as high serum ferritin levels and systemic score values. The presence of lymphadenopathy and abdominal pain was associated with MAS occurrence. (First Release April 15 2018; J Rheumatol 2018;45:864-72; doi:10.3899/jrheum.170955)
\end{abstract}

Key Indexing Terms:

ADULT ONSET STILL DISEASE SURVIVAL
FERRITIN
MACROPHAGE ACTIVATION SYNDROME HYPERFERRITINEMIC SYNDROME
From the Rheumatology Section, Department of Biotechnological and Applied Clinical Sciences, University of L'Aquila, L'Aquila; Rheumatology Section, Department of Clinical and Experimental Medicine, Second University of Naples, Naples; Rheumatology Section, Department of Internal Medicine, University of Palermo, Palermo; Department of Experimental and Clinical Medicine, University of Florence, Florence; Department of Health Sciences, University of Catanzaro "Magna Graecia," Catanzaro; Systemic Rheumatic and Autoimmune Diseases Unit, Department of Biomedical Sciences and Human Oncology, University of Bari Medical School, Bari, Italy.

P. Ruscitti, MD, Rheumatology Section, Department of Biotechnological and Applied Clinical Sciences, University of L'Aquila; D. Iacono, MD, Rheumatology Section, Department of Clinical and Experimental Medicine, Second University of Naples; F. Ciccia, MD, PhD, Rheumatology Section, Department of Internal Medicine, University of Palermo; G. Emmi, MD, PhD, Department of Experimental and Clinical Medicine, University of Florence; P. Cipriani, $M D, P h D$, Rheumatology Section, Department of Biotechnological and Applied Clinical Sciences, University of L'Aquila; R.D. Grembiale, MD, Department of Health Sciences, University of Catanzaro "Magna Graecia”; F. Perosa, MD, PhD, Systemic Rheumatic and Autoimmune Diseases Unit, Department of Biomedical Sciences and Human Oncology, University of Bari Medical
School; L. Emmi, MD, Systemic Rheumatic and Autoimmune Diseases Unit, Department of Biomedical Sciences and Human Oncology, University of Bari Medical School; G. Triolo, MD, Rheumatology Section, Department of Internal Medicine, University of Palermo; R. Giacomelli, $M D, P h D$, Rheumatology Section, Department of Biotechnological and Applied Clinical Sciences, University of L'Aquila; G. Valentini, MD, Rheumatology Section, Department of Clinical and Experimental Medicine, Second University of Naples.

Address correspondence to Dr. P. Ruscitti, Department of Biotechnological and Applied Clinical Sciences, Rheumatology Unit, School of Medicine, University of L'Aquila, Delta 6 Building, Via dell'Ospedale, 67100, L'Aquila, Italy. E-mail: pieroruscitti@live.com, piero.ruscitti@graduate.univaq.it

Accepted for publication January 15, 2018.

Adult-onset Still disease (AOSD) is a rare inflammatory disease affecting young adults, characterized by high spiking fevers, arthritis, and severe multivisceral involvement requiring immunosuppressive treatments ${ }^{1,2,3,4,5,6}$. Other usual clinical features include sore throat, elevated liver enzymes, 
lymphadenopathy, hepatosplenomegaly, and serositis ${ }^{7,8}$. During AOSD, laboratory tests show high levels of both erythrocyte sedimentation rate (ESR) and C-reactive protein (CRP), as well as of serum ferritin ${ }^{9,10}$. Patients with AOSD may experience severe complications such as macrophage activation syndrome (MAS), thrombotic thrombocytopenic purpura, and respiratory distress syndrome, leading to a decreased life expectancy ${ }^{11,12}$. MAS is defined as a reactive form of hemophagocytic lymphohistiocytosis, complicating different rheumatic diseases, mainly systemic-onset juvenile idiopathic arthritis, in which it is a major cause of mortality ${ }^{12}$. In patients with AOSD, MAS triggering factors are infections, drugs, and flares of underlying diseases, leading to a massive and uncontrollable production/release of cytokines from the immune system ${ }^{13,14}$. MAS clinical features include continuous high fever, hepatosplenomegaly, and histopathological evidence of hemophagocytes by activated macrophages, typically in bone marrow $(\mathrm{BM})^{13,14}$. This syndrome is one of the most critical clinical disorders in adults, evolving into multiple organ failure and showing unfavorable outcomes in a large percentage of patients ${ }^{15}$. In MAS, high levels of inflammatory markers and serum ferritin may be observed $^{13,14,15}$. In previous years, a possible pathogenic role of hyperferritinemia in these patients has been proposed ${ }^{16,17}$.

Although MAS is one of the leading causes of mortality in patients with AOSD, there are only a few studies that have tried to elucidate possible predictive factors of its development; these studies were mainly derived from single-center cohorts and based on a limited number of patients ${ }^{18,19,20}$. We aimed to investigate those AOSD clinical characteristics at the time of diagnosis that may be considered predictive for MAS occurrence, and to understand whether this complication may be associated with a worse outcome.

\section{MATERIALS AND METHODS}

Study design and patients. In our study, we analyzed clinical features and outcome in a multicenter Gruppo Italiano di Ricerca in Reumatologia Clinica e Sperimentale cohort of patients with AOSD. We performed a retrospective analysis of patients prospectively followed in tertiary Rheumatologic or Rare Diseases centers between January 2001 and December 2016. At baseline and during the followup, each patient was investigated for occurrence of MAS.

The local ethics committee approved the study protocol (ASL1 Avezzano-Sulmona-L'Aquila, protocol no. 0139815/16) and it has been performed according to the Good Clinical Practice guidelines and the Declaration of Helsinki.

Definition of cases. To be included in the analysis, all patients with AOSD had to fulfill the diagnostic criteria proposed by Yamaguchi, et $a l^{21}$. MAS diagnosis was defined according to the diagnostic criteria proposed by the Histiocyte Society 22,23 , and/or by Fardet, et $a l^{24}$, and/or Batu, et $a l^{25}$ for the assessment of MAS in rheumatic diseases. First, the assessment at baseline excluded potential mimickers, including infections, cancers, and other autoimmune or autoinflammatory diseases. We excluded infections by blood cultures, and in patients with MAS, BM cultures, serology and PCR analyses, chest radiographs, and abdominal echography. We evaluated possible differential diagnoses with malignancies by chest radiographs and abdominal echography, and blood samples. Despite these examinations, in the case of further suspicion of malignancy, we used computed tomography (CT) and/or positron emission tomography/CT. For patients with possible hematologic cancers, we also performed BM examination and lymph node biopsy. Autoimmune diseases were excluded by blood tests, antinuclear antibodies, anticitrullinated peptide antibodies, rheumatoid factor, and antineutrophil cytoplasmic antibodies; additionally, for the exclusion of systemic vasculitides, we included tissue biopsy and arteriography in our investigation. Finally, we evaluated possible differential diagnoses with autoinflammatory diseases by the execution of gene analyses and clinical evaluation.

Clinical assessment. The presence of the following clinical features at the time of diagnosis were recorded: spiking or continuous fever, typical rash, arthralgia or arthritis, myalgia, lymphadenopathy, sore throat, splenomegaly, hepatomegaly or abnormal liver function tests, abdominal pain, sore throat, weight loss, and gastrointestinal symptoms. The diagnosis of pleural effusion or pleuritis, and lung parenchymal involvement was made by a chest radiograph or CT scan. After clinical examinations and chest radiographs, patients with clinical suspicion of pericarditis underwent echocardiography. Taking these features together, each patient was also assessed for the systemic score proposed by Pouchot, et al for $\mathrm{AOSD}^{26}$. This score assigns 1 point to each of 12 manifestations: fever, typical rash, pleuritis, pneumonia, pericarditis, hepatomegaly or abnormal liver function tests, splenomegaly, lymphadenopathy, leukocytosis $>15,000 / \mathrm{mm}^{3}$, sore throat, myalgia, and abdominal pain (max score: 12 points). ESR, CRP, and serum ferritin levels were also registered. The presence of comorbidities was investigated at the time of first observation, defined as coexisting medical conditions that were distinct from the principal diagnosis for which the patient was enrolled in our study.

The therapeutic strategies were recorded. Treatment regimens used at the time of diagnosis and during followup were categorized into 4 groups, based on the treatment regimen administered to the patient for the longest time period: (1) low/medium dose of steroids: $\leq 0.5 \mathrm{mg} / \mathrm{kg} / \mathrm{day}$ of prednisone; (2) high dose of steroids: $>0.5 \mathrm{mg} / \mathrm{kg} / \mathrm{day}$ of prednisone; (3) combination therapy with steroids + synthetic disease-modifying antirheumatic drugs (sDMARD); and (4) steroids + biologic drugs \pm SDMARD.

According to the disease course at the last scheduled visit, patients were divided into 4 groups as described by Cush, et $a l^{27}: 3$ clinical patterns (monocyclic, polycyclic, chronic) and death. A monocyclic course was defined as a single episode for $>2$ months but $<1$ year, followed by sustained remission through the entire followup period. A polycyclic course was characterized by recurrent systemic flares with remission between flares. A chronic course was defined as $\geq 1$ episode of persistent symptoms lasting $>1$ year. Patients who were diagnosed with AOSD and who died during followup were placed in the death group. AOSD-related death was defined as death associated with AOSD or its complications during the followup.

In addition, at the time of diagnosis and during followup, each patient was assessed for the presence of AOSD-related complications including MAS, thrombotic thrombocytopenic purpura, thrombotic microangiopathy, disseminated intravascular coagulopathy, respiratory distress syndrome, diffuse alveolar hemorrhage, pulmonary arterial hypertension, myocarditis, tamponade, constrictive pericarditis, endocarditis, shock, multiple organ failure, fulminant hepatitis, and amyloidosis, as suggested by available literature ${ }^{28}$.

Statistical analysis. An observational design was set to provide a risk model profile for patients with MAS. The presence of the following clinical features was recorded and progressively added to the analyses: fever, typical rash, pleuritis, pneumonia, pericarditis, hepatomegaly or abnormal liver function tests, splenomegaly, lymphadenopathy, leukocytosis $>15,000 / \mathrm{mm}^{3}$, sore throat, myalgia and abdominal pain, systemic score, ESR, CRP, and serum ferritin levels. Therapeutic strategies were also analyzed. The preliminary statistical analysis provided descriptive statistics. Continuous variables that were normally distributed were expressed as mean $\pm \mathrm{SD}$, whereas continuous variables that were not normally distributed were expressed as median (interquartile range; IQR). To compare the clinical characteristics between patients who had MAS at first admission or who developed it during

Personal non-commercial use only. The Journal of Rheumatology Copyright $\subset$ C 2018. All rights reserved. 
followup with the remaining patients, the $\mathrm{T}$ test was used for all the continuous variables that were normally distributed, whereas Mann-Whitney U Test was used for those not normally distributed. The chi-squared test was used for all the categorical variables. Bonferroni correction was systematically applied to counteract the problem of multiple comparisons; thus, statistical significance for these multiple comparisons was expressed by $\mathrm{p}$ value $<0.005$.

Receiver-operating characteristic (ROC) curves were performed to evaluate the possible role of serum ferritin and systemic scores in characterizing patients with MAS at the first admission or those who developed it during followup. The best cutoff for ROC curves was calculated with the Youden's index. Regression analyses were performed assessing the statistical significance of possible clinical and laboratory associations on the likelihood of MAS occurrence. The purposeful selection process of covariates started by a univariate analysis of each variable; any variable having a significant univariate test was selected as a possible candidate for the multivariate analysis. Conversely, covariates were removed from the model if they were nonsignificant. At the end of this multistep process of deleting and refitting, the multivariate model was built, and OR estimations of significant associations with MAS were provided. Finally, Kaplan-Meier curves were plotted to determine the rates of survival of patients with AOSD who developed MAS when compared with patients who did not develop MAS. The difference between curves was determined by the log-rank (Mantel-Cox) test.

Because of the relatively simple design of our study, we had a very low percentage of missing data; patients were removed from the study if they had missing values that were meaningful for analysis. The Statistics Package for Social Sciences (SPSS version 17.0, SPSS Inc.) was used for all analyses.

\section{RESULTS}

Clinical characteristics of the evaluated patients at time of diagnosis. Table 1 and Table 2 list the demographic and clinical features of the 119 investigated patients [71 men, 48 women, age at diagnosis: median 44 (range 18-79)]. Originally, 123 patients were assessed, but 4 patients were not included in the study because of missing values that were meaningful for analysis. Briefly, all 119 evaluated patients experienced fever (100\%), 104 patients $(87.4 \%)$ showed joint involvement, 90 patients displayed rash $(75.6 \%), 72$ patients $(60.5 \%)$ hepatic involvement, and 85 patients $(71.4 \%)$ splenomegaly. The systemic score resulted in a median of 6 (IQR 5.0-7.0). In our cohort, a marked increase of inflammatory markers at the time of diagnosis was observed: serum ferritin levels $1200 \mathrm{ng} / \mathrm{ml}(727.5-29,500)$; ESR 69.9 \pm 26.2 $\mathrm{mm} / \mathrm{h}$; and CRP $50 \mathrm{mg} / \mathrm{l}(20.0-120.0)$. We found that at the time of diagnosis, 38 patients $(31.9 \%)$ presented with $\geq 1$ comorbidity. In fact, 24 patients were affected by systemic arterial hypertension, 10 by dyslipidemia, 10 by thyroid

Table 1. Comparison of clinical features between patients with and without MAS, at the time of diagnosis. Values are $\mathrm{n}(\%)$ or mean $\pm \mathrm{SD}$ unless otherwise specified.

\begin{tabular}{|c|c|c|c|}
\hline Characteristics & Without MAS & Presenting with MAS & $\mathrm{p}^{*}$ \\
\hline Women (men), $\mathrm{n}$ & $42(65)$ & $6(6)$ & 0.472 \\
\hline Age, yrs & $44.8 \pm 15.9$ & $45.1 \pm 17.7$ & 0.677 \\
\hline \multicolumn{4}{|l|}{ Clinical features } \\
\hline Fever & $107(100)$ & $12(100)$ & - \\
\hline Arthritis & $94(87.8)$ & $10(83.3)$ & 0.655 \\
\hline Myalgia & $64(59.8)$ & $9(75)$ & 0.306 \\
\hline Rash & $81(75.7)$ & $9(75)$ & 0.957 \\
\hline Splenomegaly & $74(69.1)$ & $11(91.6)$ & 0.102 \\
\hline Liver involvement & $61(57)$ & $11(91.6)$ & 0.02 \\
\hline Lymphadenopathy & $54(50.4)$ & $10(83.3)$ & 0.026 \\
\hline Sore throat & $60(56.1)$ & $10(83.3)$ & 0.069 \\
\hline Lung involvement & $13(12.1)$ & $2(16.6)$ & 0.655 \\
\hline Pericarditis & $18(16.8)$ & $3(25)$ & 0.481 \\
\hline Pleurisy & $15(14.0)$ & $5(41.7)$ & 0.083 \\
\hline Abdominal pain & $16(14.9)$ & $4(33.3)$ & 0.106 \\
\hline Weight loss & $2(1.9)$ & $5(41.7)$ & $<0.0001$ \\
\hline Systemic score, median (IQR) & $5(4.0-7.0)$ & $8(6.2-8.7)$ & 0.002 \\
\hline Comorbidities & $32(29.9)$ & $6(50)$ & 0.330 \\
\hline \multicolumn{4}{|l|}{ Laboratory markers } \\
\hline Leukocytosis $>15,000 / \mathrm{mm}^{3}$ & $49(45.8)$ & $5(41.7)$ & 0.785 \\
\hline Serum ferritin, ng/ml, median (IQR) & $1120(647.0-2393.5)$ & $3510(1586.2-7444.0)$ & 0.001 \\
\hline $\mathrm{ESR}, \mathrm{mm} / \mathrm{h}$ & $69.5 \pm 25.9$ & $74.5 \pm 30.3$ & 0.525 \\
\hline $\mathrm{CRP}, \mathrm{mg} / \mathrm{l}$, median (IQR) & $51[19.7-119.0]$ & $45[18.7-114.0]$ & 0.261 \\
\hline \multicolumn{4}{|l|}{ Therapeutic strategies } \\
\hline Low-dose steroid monotherapy & $7(6.5)$ & 0 & 0.361 \\
\hline High-dose steroid monotherapy & $41(38.3)$ & $5(41.7)$ & 0.821 \\
\hline Combination therapy, steroids + sDMARD & $29(27.1)$ & $5(41.7)$ & 0.290 \\
\hline \multicolumn{4}{|l|}{ Combination therapy, steroids + biologics } \\
\hline \pm sDMARD & $30(28.1)$ & $2(16.6)$ & 0.400 \\
\hline
\end{tabular}

\footnotetext{
* Statistical significance was expressed by $\mathrm{p}$ value $<0.005$. Values in bold face indicate statistically significant results. MAS: macrophage activation syndrome; ESR: erythrocyte sedimentation rate; CRP: C-reactive protein; sDMARD: synthetic disease-modifying antirheumatic drugs; IQR: interquartile range.
} 
Table 2. Baseline clinical features comparison between patients with and without MAS during the followup. Values are $\mathrm{n}(\%)$ or mean \pm SD unless otherwise specified.

\begin{tabular}{|c|c|c|c|}
\hline Characteristics & Without MAS & Developing MAS & $\mathrm{p}^{*}$ \\
\hline Women (men), n & $41(61)$ & $1(4)$ & 0.367 \\
\hline Age, yrs & $44.2 \pm 15.7$ & $57.0 \pm 18.4$ & 0.080 \\
\hline \multicolumn{4}{|l|}{ Clinical features } \\
\hline Fever & $102(100)$ & $5(100)$ & - \\
\hline Arthritis & $91(89.2)$ & $3(60)$ & 0.051 \\
\hline Myalgia & $59(57.8)$ & $5(100)$ & 0.060 \\
\hline Rash & $78(76.5)$ & $3(60)$ & 0.402 \\
\hline Splenomegaly & $69(67.6)$ & $5(100)$ & 0.126 \\
\hline Liver involvement & $57(55.9)$ & $4(80)$ & 0.288 \\
\hline Lymphadenopathy & $48(47.1)$ & $5(100)$ & 0.021 \\
\hline Sore throat & $55(53.9)$ & $5(100)$ & 0.043 \\
\hline Lung involvement & $11(10.8)$ & $2(40)$ & 0.051 \\
\hline Pericarditis & $16(15.7)$ & $2(40)$ & 0.156 \\
\hline Pleurisy & $14(13.7)$ & $1(20)$ & 0.693 \\
\hline Abdominal pain & $13(12.7)$ & $3(60)$ & 0.004 \\
\hline Weight loss & $2(1.9)$ & 0 & 0.569 \\
\hline Systemic score, median (IQR) & $5(4-7)$ & $8(7-9.5)$ & 0.003 \\
\hline Comorbidities & $30(30.6)$ & $2(40)$ & 0.299 \\
\hline \multicolumn{4}{|l|}{ Laboratory markers } \\
\hline Leukocytosis $>15,000 / \mathrm{mm}^{3}$ & $48(47.1)$ & $1(20)$ & 0.236 \\
\hline Serum ferritin, ng/ml, median (IQR) & $090(623.0-2000.0)$ & $4453(1515.0-8441.0)$ & 0.013 \\
\hline $\mathrm{ESR}, \mathrm{mm} / \mathrm{h}$ & $69.2 \pm 25.5$ & $75.4 \pm 34.8$ & 0.602 \\
\hline CRP, mg/l, median (IQR) & $90(20.0-110.0)$ & $120(40.0-180.0)$ & 0.082 \\
\hline \multicolumn{4}{|l|}{ Therapeutic strategies } \\
\hline Low-dose steroid monotherapy & $7(6.9)$ & 0 & 0.545 \\
\hline High-dose steroid monotherapy & $39(38.2)$ & $2(40)$ & 0.937 \\
\hline Combination therapy, steroids + sDMARD & $28(27.4)$ & $1(20)$ & 0.714 \\
\hline \multicolumn{4}{|l|}{ Combination therapy, steroids + biologics } \\
\hline$\pm \mathrm{sDMARD}$ & $28(27.4)$ & $2(40)$ & 0.542 \\
\hline
\end{tabular}

* Statistical significance was expressed by $\mathrm{p}$ value $<0.005$. Values in bold face indicate statistically significant results. MAS: macrophage activation syndrome; ESR: erythrocyte sedimentation rate; CRP: C-reactive protein; sDMARD: synthetic disease-modifying antirheumatic drugs; IQR: interquartile range.

disease, 10 by type 2 diabetes, 4 by ischemic cardiomyopathy, and 2 by atrial fibrillation.

Seven patients $(5.9 \%)$ were treated or were being treated at admission with low-dose steroid monotherapy $(\leq 5 \mathrm{mg} / \mathrm{d})$, $46(38.6 \%)$ with high-dose steroid monotherapy, 34 (28.6\%) with combination therapy steroids + sDMARD, and 32 (26.9\%) with combination therapy steroids + biologic drugs \pm sDMARD. Analyzing sDMARD-treated patients, we observed that cyclosporine and methotrexate were administered, whereas no patient was treated with cytotoxic drugs such as etoposide.

MAS occurrence: presence at the time of diagnosis or development during the followup. In our study, we reported that 17 patients had MAS triggered by disease flare and/or severe infections. All patients with MAS underwent BM aspirate and/or biopsy. Peripheral blood cytopenia, liver abnormalities, and high levels of triglycerides during the full-blown syndrome were observed in these patients (white blood cell count $5.36 \pm 2.4610^{3} / \mathrm{ml}$, platelet count $65.32 \pm 36.27$ $10^{3} / \mathrm{ml}$, red blood cell count $3.73 \pm 1.1810^{3} / \mathrm{ml}$, hemoglobin $7.89 \pm 2.01 \mathrm{~g} / \mathrm{dl}$, triglycerides $213.12 \pm 64.56 \mathrm{mg} / \mathrm{dl}$, aspartate aminotransferase $82.24 \pm 16.87 \mathrm{IU} / \mathrm{l}$, and alanine aminotransferase $164.36 \pm 54.31 \mathrm{IU} / \mathrm{l})$.

Twelve out of the 119 patients presented with definite MAS at first admission to their respective center. Clinical and laboratory features of these patients presenting with MAS are listed in Table 1 . The serum ferritin levels were significantly higher in patients presenting with MAS when compared with those without MAS at the time of diagnosis $[3510 \mathrm{ng} / \mathrm{ml}$ (1586.2-7444.0) vs $1120 \mathrm{ng} / \mathrm{ml}(647.0-2393.5)$, respectively, $\mathrm{p}=0.001]$. Similarly, the values of systemic score were significantly higher in patients presenting with MAS [8 $(6.2-8.7)$ vs $5(4.0-7.0)$, respectively, $\mathrm{p}=0.002]$. Using ROC analyses, serum ferritin $\geq 1320.5 \mathrm{ng} / \mathrm{ml}$ identified patients with MAS at admission, with a sensitivity of $91 \%$ and a specificity of $60 \%$, whereas a systemic score $\geq 6.5$ identified patients with MAS at admission with a sensitivity of $75 \%$ and a specificity of $68 \%$.

Six patients developed AOSD-related complications during followup. Specifically, 1 patient developed myocarditis, whereas 5 patients developed MAS. Clinical and laboratory features during followup of patients without MAS

Personal non-commercial use only. The Journal of Rheumatology Copyright $\odot$ 2018. All rights reserved. 
or who developed MAS are listed in Table 2. At the time of diagnosis, the 5 patients who developed MAS during followup differed from the remaining 102 by the prevalence of abdominal pain $(\mathrm{p}=0.004)$. In patients who developed MAS during followup, the serum ferritin levels at the time of diagnosis were higher than in patients who did not develop this complication [4453 ng/ml (1515.0-8441.0) vs 1090 $\mathrm{ng} / \mathrm{ml}$ (623.0-2000.0), respectively]. Similarly, the systemic scores at the time of diagnosis were higher in patients who developed MAS versus those without it [8 (7.0-9.5) vs 5 $(4.0-7.0), \mathrm{p}=0.003]$. ROC analyses determined that the best serum ferritin level cutoff that discriminated patients who developed MAS from those who did not was $1484.5 \mathrm{ng} / \mathrm{ml}$ (sensitivity $80 \%$, specificity $63 \%$ ) and the best systemic score cutoff was $\geq 7.5$ (sensitivity $80 \%$, specificity $83 \%$ ).

Predictive factors of MAS occurrence. Regression analyses were performed assessing the statistical significance of possible clinical and laboratory associations on the likelihood of MAS occurrence (Table 3). Because of the rarity of the disease and the relatively low number of patients experiencing MAS in our cohort, patients showing MAS at first observation and patients who developed MAS during followup were analyzed together in our analysis, which aimed to evaluate predictive factors of MAS occurrence. In univariate analyses, evidence of these factors was significantly associated with MAS occurrence: liver involvement (OR 5.92, 95\% CI 1.29-27.24, $\mathrm{p}=0.022$ ), lymphadenopathy (OR 8.43, 95\% CI 1.83-38.80, $\mathrm{p}=0.006$ ), sore throat (OR $6.41,95 \%$ CI $1.39-29.49, \mathrm{p}=0.017)$, and abdominal pain (OR 4.79, 95\% CI 1.55-14.80, $\mathrm{p}=0.006$ ). Conversely, these were not associated with MAS: sex, age, other recorded clinical features, ESR and CRP values, presence of comorbidities, and the different therapeutic strategies. In the multivariate analysis, the evidence of lymphadenopathy (OR 7.22, 95\% CI 1.49-34.97, $\mathrm{p}=0.014$ ) and abdominal pain (OR 4.36, $95 \%$ CI $1.24-15.39, \mathrm{p}=0.022)$ were retained in the model as predictive of MAS occurrence.

Analysis of survival in patients experiencing MAS. The patients were followed for a minimum of 0.6 years to a maximum of 16 years from admission (25.6 patients/ followup yr). During the followup period, 100 patients (84\%) experienced a favorable outcome, 36 patients (30.3\%) showed a monocyclic pattern, 37 patients $(31.1 \%)$ a polycyclic pattern, and 27 patients $(22.7 \%)$ a chronic pattern (Table 4). Nineteen patients died during the followup period. Specifically, 12 patients died from uncontrollable MAS with

Table 3. Regression analyses to define factors associated with MAS in patients with AOSD at the time of diagnosis.

\begin{tabular}{|c|c|c|c|}
\hline Variables & OR & $95 \% \mathrm{CI}$ & $\mathrm{p}^{*}$ \\
\hline \multicolumn{4}{|l|}{ Univariate analyses } \\
\hline Sex & 0.96 & $0.34-2.73$ & 0.94 \\
\hline Age & 1.02 & $0.98-1.05$ & 0.30 \\
\hline Arthritis & 0.39 & $0.11-1.42$ & 0.15 \\
\hline Myalgia & 3.40 & $0.92-12.57$ & 0.07 \\
\hline Rash & 0.74 & $0.24-2.31$ & 0.60 \\
\hline Splenomegaly & 7.65 & $0.97-60.18$ & 0.06 \\
\hline Liver involvement & 5.92 & $1.29-27.24$ & 0.022 \\
\hline Lymphadenopathy & 8.43 & $1.83-38.80$ & 0.006 \\
\hline Sore throat & 6.41 & $1.39-29.49$ & 0.017 \\
\hline Lung involvement & 2.54 & $0.70-9.19$ & 0.15 \\
\hline Pericarditis & 2.24 & $0.69-7.23$ & 0.18 \\
\hline Pleurisy & 2.62 & $0.80-8.57$ & 0.11 \\
\hline Abdominal pain & 4.79 & $1.55-14.80$ & 0.006 \\
\hline Weight loss & 1.00 & $0.99-1.01$ & 0.99 \\
\hline Comorbidities & 1.35 & $0.75-2.43$ & 0.32 \\
\hline Leukocytosis & 0.61 & $0.21-1.78$ & 0.37 \\
\hline ESR & 1.01 & $0.99-1.03$ & 0.41 \\
\hline CRP & 0.99 & $0.98-1.01$ & 0.87 \\
\hline Low-dose steroid monotherapy & 0.98 & $0.97-1.01$ & 0.99 \\
\hline High-dose steroid monotherapy & 1.13 & $0.40-3.22$ & 0.82 \\
\hline Combination therapy, steroids + sDMARD & 1.44 & $0.49-4.27$ & 0.51 \\
\hline Combination therapy, steroids + biologics \pm sDMARD & 0.81 & $0.24-2.70$ & 0.74 \\
\hline \multicolumn{4}{|l|}{ Multivariate analyses } \\
\hline Liver involvement & 3.94 & $0.80-19.41$ & 0.09 \\
\hline Lymphadenopathy & 7.22 & 1.49-34.97 & 0.014 \\
\hline Sore throat & 4.71 & $0.96-23.01$ & 0.06 \\
\hline Abdominal pain & 4.36 & $1.24-15.39$ & 0.022 \\
\hline
\end{tabular}

* Statistical significance was expressed by a p value $<0.05$. Values in bold face indicate statistically significant results. MAS: macrophage activation syndrome; AOSD: adult-onset Still disease; ESR: erythrocyte sedimentation rate; CRP: C-reactive protein; sDMARD: synthetic disease-modifying antirheumatic drugs. 
Table 4. Outcome of the evaluated patients with AOSD. Values are n (\%) unless otherwise specified.

\begin{tabular}{lc}
\hline Outcome & Patients \\
\hline Favorable outcome & $100(84)$ \\
Monocyclic course & $36(30.3)$ \\
Polycyclic course & $37(31.1)$ \\
Chronic course & $27(22.7)$ \\
Unfavorable outcome & $19(16)$ \\
AOSD-related death & $19(16)$ \\
Time of followup, yrs, median (min-max) & $3.9(0.6-16.0)$ \\
\hline
\end{tabular}

AOSD: adult-onset Still disease.

coagulation disorders, 5 from multiple organ failure with liver and kidney failures, and 2 from severe infection related to the immunosuppressive therapy. Because of the rarity of the disease and the relatively low number of patients experiencing MAS in our cohort, patients showing MAS at the first observation and patients who developed MAS during the followup were also analyzed together to minimize the possible confounding effect of the low number of patients on the statistical analyses. Nineteen patients died during followup; in 12 patients, the main cause of death was the development of MAS. In fact, the development of MAS was significantly associated with a decreased survival rate of patients ( $\mathrm{p}<0.0001$; HR 12.71, 95\% CI 11.19-14.23; Figure 1).

\section{DISCUSSION}

To our knowledge, this is the first study devoted to investigating the clinical characteristics at the time of diagnosis that may be considered predictive for MAS occurrence, and to understanding whether this complication may be associated with a decreased survival rate, in one of the largest cohorts of patients with AOSD described in literature.

The clinical features of our patients did not significantly differ from other available series already published, concerning the prevalence of fever, arthritis, and rash ${ }^{29-37}$. Our patients also showed high levels of inflammatory markers, as well as of serum ferritin, thus confirming the systemic inflammatory features of the disease $\mathrm{e}^{29,30,31,32}$.

In our AOSD cohort, 17 patients presented with MAS at the time of diagnosis or during the disease course, suggesting that the association between these 2 diseases is more frequent than that of any other adult inflammatory diseases, as well as suggesting possible overlapping pathogenic mechanisms ${ }^{28}$. In fact, MAS together with AOSD has been included under a common umbrella, the hyperferritinemic syndrome, in which the pathogenic role of ferritin has been postulated ${ }^{16,17}$.

\section{Survival Function}

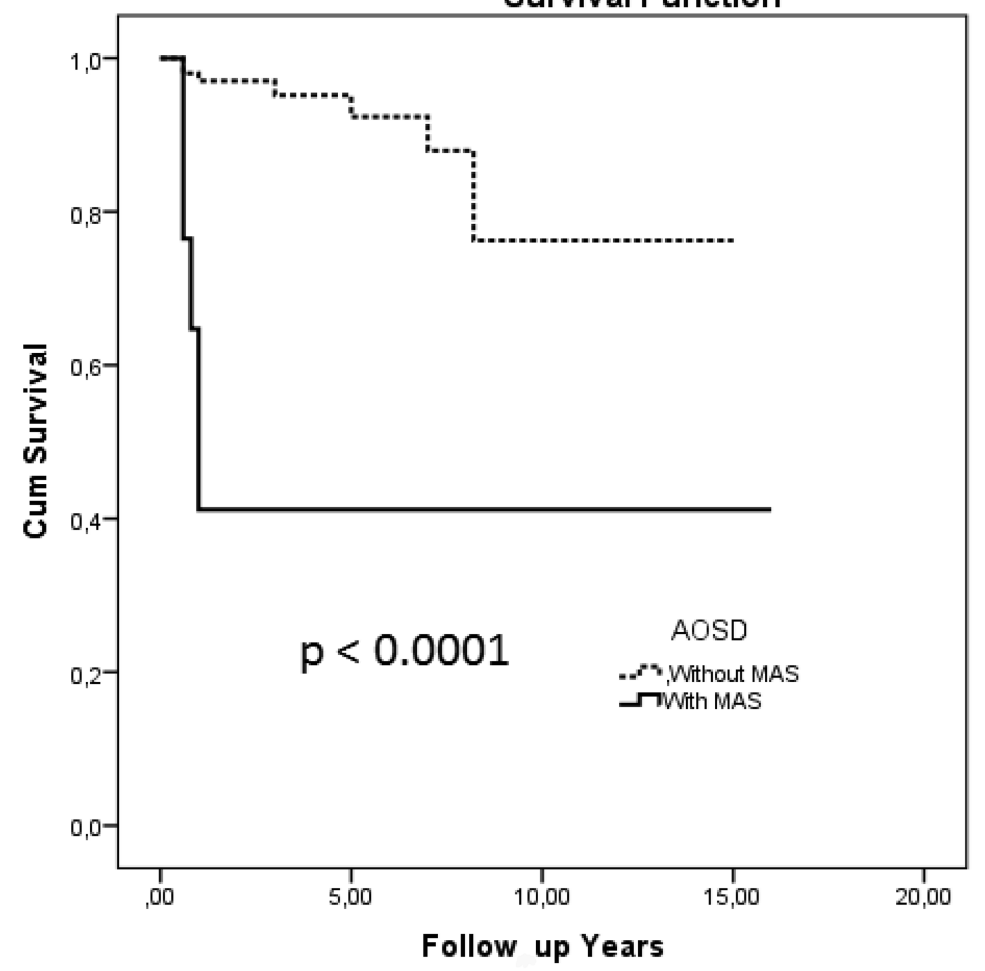

Figure 1. Kaplan-Meier curves of survival in AOSD patients with or without MAS. Nineteen patients died during the followup; the main cause of death was the development of MAS in 12 patients. The development of MAS was significantly associated with a decreased survival rate of patients with AOSD ( $<<0.0001 ;$ HR 12.71,95\% CI 11.19-14.23). AOSD: adult-onset Still disease; MAS: macrophage activation syndrome.

Personal non-commercial use only. The Journal of Rheumatology Copyright (C) 2018. All rights reserved. 
Further, it has been shown that MAS occurrence may be misdiagnosed in patients treated with immunosuppressive agents used to control AOSD clinical features ${ }^{13,14,28}$.

Of note, serum ferritin levels, systemic score values, prevalence of lymphadenopathy, and liver involvement were significantly higher at the time of diagnosis in those patients with AOSD who had MAS, identifying a subset of AOSD patients with a more aggressive disease, as previously shown ${ }^{38,39}$. We also observed that the patients experiencing MAS during the followup showed, at the time of diagnosis, increased systemic score values and higher prevalence of abdominal pain when compared with AOSD patients not developing this complication. In addition, the presence of lymphadenopathy and liver involvement was associated with occurrence of this complication. Mild to severe enlargement of lymph nodes as well as liver abnormalities were observed in patients with AOSD, not only because of a widespread accumulation of inflammatory cells in these organs but also for the occurrence of hemophagocytosis, which is characteristically observed in BM, liver, and peripheral lymphoid tissues ${ }^{13}$. Further, the presence of abdominal pain at the time of diagnosis was significantly predictive of MAS occurrence. This clinical sign is mainly related to peritoneal serositis and should be carefully evaluated in patients with $\operatorname{AOSD}^{1,13,14}$. Our results may help the clinician to better identify these 2 frequently associated conditions and to aggressively treat those patients with AOSD who are at higher risk of MAS occurrence.

In our study, a significant reduction of survival rates was observed in patients affected by MAS. The higher mortality rate observed in our study does not confirm results from other European AOSD series, in which lower mortality rates were described $^{29,30,31,32}$, but it is in parallel with published data reported on Asiatic populations ${ }^{35,36}$. MAS is generally considered one of the worst prognostic factors in AOSD, strongly associated with a high mortality rate that is probably related to comorbidities frequently observed among adults $^{14,15,35-41}$.

We did not observe any possible association between the use of different drugs and MAS development during followup. Our retrospective study was not specifically designed to analyze the possible effects of any drug in modifying patient outcomes. Future adequately powered and well-designed studies are needed to fully elucidate this issue. In addition, it has been shown in the juvenile counterpart of AOSD that although biologic agents inhibiting interleukin 1 (IL-1) or IL-6 are effective in the treatment of signs and symptoms of the disease, such drugs in clinical trials did not provide full protection against MAS development, suggesting the presence of further mechanisms in the pathogenesis of this complication $42,43,44,45$.

As observed in any retrospective study, different limitations may exist. Because of the multicenter and retrospective design, some biases such as selection and recall are possible.
In addition, different diagnostic criteria were used for the diagnosis according to data availability, limiting the external validity of our findings. However, it must be pointed out that both AOSD and MAS are very rare diseases, and designing and carrying out prospective and adequately powered studies is quite difficult, as reported for other infrequent complications in rheumatic diseases $46,47,48,49,50$

MAS occurrence significantly decreased the survival rate in patients with AOSD. As expected, patients with MAS at the time of diagnosis presented higher levels of serum ferritin, elevated systemic score, prevalence of lymphadenopathy, and liver involvement. The presence of lymphadenopathy and abdominal pain was associated with occurrence of this complication. The results of our study, which determined the clinical characteristics at the time of diagnosis that may be predictive for MAS occurrence, may help in the clinical setting to better identify those patients needing a more aggressive treatment to avoid the life-threatening evolution of AOSD.

\section{ACKNOWLEDGMENT}

The authors thank Federica Sensini for her technical assistance.

\section{REFERENCES}

1. Gerfaud-Valentin M, Jamilloux Y, Iwaz J, Sève P. Adult-onset Still's disease. Autoimmun Rev 2014;13:708-22.

2. Jamilloux Y, Gerfaud-Valentin M, Henry T, Sève P. Treatment of adult-onset Still's disease: a review. Ther Clin Risk Manag 2014;11:33-43.

3. Cipriani P, Ruscitti P, Carubbi F, Liakouli V, Giacomelli R. Methotrexate: an old new drug in autoimmune disease. Expert Rev Clin Immunol 2014;10:1519-30.

4. Ruscitti P, Ursini F, Cipriani P, De Sarro G, Giacomelli R. Biologic drugs in adult onset Still's disease: a systematic review and meta-analysis of observational studies. Expert Rev Clin Immunol 2017;13:1089-97.

5. Cipriani P, Ruscitti P, Carubbi F, Pantano I, Liakouli V, Berardicurti O, et al. Tocilizumab for the treatment of adult-onset Still's disease: results from a case series. Clin Rheumatol 2014;33:49-55.

6. Colafrancesco S, Priori R, Valesini G, Argolini L, Baldissera E, Bartoloni E, et al. Response to interleukin-1 inhibitors in 140 Italian patients with adult-onset still's disease: a multicentre retrospective observational study. Front Pharmacol 2017;8:369.

7. Sfriso P, Priori R, Valesini G, Rossi S, Montecucco CM, D'Ascanio A, et al. Adult-onset Still's disease: an Italian multicentre retrospective observational study of manifestations and treatments in 245 patients. Clin Rheumatol 2016;35:1683-9.

8. Ruscitti P, Ciccia F, Cipriani P, Guggino G, Di Benedetto P, Rizzo A, et al. The CD68(+)/H-ferritin(+) cells colonize the lymph nodes of the patients with adult onset Still's disease and are associated with increased extracellular level of $\mathrm{H}$-ferritin in the same tissue: correlation with disease severity and implication for pathogenesis. Clin Exp Immunol 2016;183:397-404.

9. Mitrovic S, Fautrel B. New markers for adult-onset Still's disease. Joint Bone Spine 2017 May 18 (Epub ahead of print).

10. Mehta B, Efthimiou P. Ferritin in adult-onset still's disease: just a useful innocent bystander? Int J Inflam 2012;2012:298405.

11. Ahn SS, Yoo BW, Jung SM, Lee SW, Park YB, Song JJ. Application of the 2016 EULAR/ACR/PRINTO classification criteria for macrophage activation syndrome in patients with adult-onset still disease. J Rheumatol 2017;44:996-1003.

Personal non-commercial use only. The Journal of Rheumatology Copyright (C) 2018. All rights reserved 
12. Minoia F, Davì S, Horne A, Bovis F, Demirkaya E, Akikusa J, et al; Pediatric Rheumatology International Trials Organization; Childhood Arthritis and Rheumatology Research Alliance; Pediatric Rheumatology Collaborative Study Group; Histiocyte Society. Dissecting the heterogeneity of macrophage activation syndrome complicating systemic juvenile idiopathic arthritis. J Rheumatol 2015;42:994-1001.

13. Ramos-Casals M, Brito-Zerón P, López-Guillermo A, Khamashta MA, Bosch X. Adult haemophagocytic syndrome. Lancet 2014;383:1503-16.

14. Grom AA, Horne A, De Benedetti F. Macrophage activation syndrome in the era of biologic therapy. Nat Rev Rheumatol 2016;12:259-68.

15. Ruscitti P, Cipriani P, Ciccia F, Masedu F, Liakouli V, Carubbi F, et al. Prognostic factors of macrophage activation syndrome, at the time of diagnosis, in adult patients affected by autoimmune disease: analysis of 41 cases collected in 2 rheumatologic centers. Autoimmun Rev 2017;16:16-21.

16. Ruscitti P, Cipriani P, Di Benedetto P, Ciccia F, Liakouli V, Carubbi $\mathrm{F}$, et al. Increased level of $\mathrm{H}$-ferritin and its imbalance with L-ferritin, in bone marrow and liver of patients with adult onset Still's disease, developing macrophage activation syndrome, correlate with the severity of the disease. Autoimmun Rev 2015;14:429-37.

17. Rosário C, Zandman-Goddard G, Meyron-Holtz EG, D’Cruz DP, Shoenfeld Y. The hyperferritinemic syndrome: macrophage activation syndrome, Still's disease, septic shock and catastrophic antiphospholipid syndrome. BMC Med $2013 ; 11: 185$.

18. Kong XD, Xu D, Zhang W, Zhao Y, Zeng X, Zhang F. Clinical features and prognosis in adult-onset Still's disease: a study of 104 cases. Clin Rheumatol 2010;29:1015-9.

19. Kim YJ, Koo BS, Kim YG, Lee CK, Yoo B. Clinical features and prognosis in 82 patients with adult-onset Still's disease. Clin Exp Rheumatol 2013;32:28-33.

20. Fautrel B, Le Moël G, Saint-Marcoux B, Taupin P, Vignes S, Rozenberg S, et al. Diagnostic value of ferritin and glycosylated ferritin in adult onset Still's disease. J Rheumatol 2001;28:322-9.

21. Yamaguchi M, Ohta A, Tsunematsu T, Kasukawa R, Mizushima Y, Kashiwagi H, et al. Preliminary criteria for classification of adult Still's disease. J Rheumatol 1992;19:424-30.

22. Henter JI, Elinder G, Ost A. Diagnostic guidelines for hemophagocytic lymphohistiocytosis. The FHL Study Group of the Histiocyte Society. Semin Oncol 1991;18:29-33.

23. Henter JI, Horne A, Aricó M, Egeler RM, Filipovich AH, Imashuku S, et al. HLH-2004: Diagnostic and therapeutic guidelines for hemophagocytic lymphohistiocytosis. Pediatr Blood Cancer 2007;48:124-31.

24. Fardet L, Galicier L, Lambotte O, Marzac C, Aumont C, Chahwan $\mathrm{D}$, et al. Development and validation of the HScore, a score for the diagnosis of reactive hemophagocytic syndrome. Arthritis Rheumatol 2014;66:2613-20.

25. Batu ED, Erden A, Seyhoğlu E, Kilic L, Büyükasık Y, Karadag O, et al. Assessment of the HScore for reactive haemophagocytic syndrome in patients with rheumatic diseases. Scand J Rheumatol 2017;46:44-8.

26. Pouchot J, Sampalis JS, Beaudet F, Carette S, Décary F, Salusinsky-Sternbach M, et al. Adult Still's disease: manifestations, disease course, and outcome in 62 patients. Medicine 1991; 70:118-136.

27. Cush JJ, Medsger TA Jr, Christy WC, Herbert DC, Cooperstein LA. Adult-onset Still's disease. Clinical course and outcome. Arthritis Rheum 1987;30:186-94.

28. Efthimiou P, Kadavath S, Mehta B. Life-threatening complications of adult-onset Still's disease. Clin Rheumatol 2014;33:305-14.

29. Fautrel B, Zing E, Golmard JL, Le Moel G, Bissery A, Rioux C, et al. Proposal for a new set of classification criteria for adult-onset still disease. Medicine 2002;81:194-200.

30. Colina M, Zucchini W, Ciancio G, Orzincolo C, Trotta F, Govoni M. The evolution of adult-onset Still disease: an observational and comparative study in a cohort of 76 Italian patients. Semin Arthritis Rheum 2011;41:279-85.

31. Gerfaud-Valentin M, Maucort-Boulch D, Hot A, Iwaz J, Ninet J, Durieu I, et al. Adult-onset Still disease: manifestations, treatments, outcome, and prognostic factors in 57 patients. Medicine 2014;93:91-9.

32. Kalyoncu U, Solmaz D, Emmungil H, Yazici A, Kasifoglu T, Kimyon G, et al. Response rate of initial conventional treatments, disease course, and related factors of patients with adult-onset Still's disease: data from a large multicenter cohort. J Autoimmun 2016;69:59-63.

33. Cozzi A, Papagrigoraki A, Biasi D, Colato C, Girolomoni G. Cutaneous manifestations of adult-onset Still's disease: a case report and review of literature. Clin Rheumatol 2016;35:1377-82.

34. Ruscitti P, Cipriani P, Ciccia F, Di Benedetto P, Liakouli V, Berardicurti O, et al. H-ferritin and CD68(+) /H-ferritin(+) monocytes/macrophages are increased in the skin of adult-onset Still's disease patients and correlate with the multi-visceral involvement of the disease. Clin Exp Immunol 2016;186:30-8.

35. Kim HA, Sung JM, Suh CH. Therapeutic responses and prognosis in adult-onset Still's disease. Rheumatol Int 2012;32:1291-8.

36. Zeng T, Zou YQ, Wu MF, Yang CD. Clinical features and prognosis of adult-onset still's disease: 61 cases from China. J Rheumatol 2009;36:1026-31.

37. Giacomelli R, Gorla R, Trotta F, Tirri R, Grassi W, Bazzichi L, et al. Quality of life and unmet needs in patients with inflammatory arthropathies: results from the multicentre, observational RAPSODIA study. Rheumatology 2015;54:792-7.

38. Ruscitti P, Cipriani P, Masedu F, Iacono D, Ciccia F, Liakouli V, et al. Adult-onset Still's disease: evaluation of prognostic tools and validation of the systemic score by analysis of 100 cases from three centers. BMC Med 2016;14:194.

39. Guilpain P, Le Quellec A. About the complexity of adult onset Still's disease... and advances still required for its management. BMC Med 2017;15:5.

40. Ruscitti P, Rago C, Breda L, Cipriani P, Liakouli V, Berardicurti O, et al. Macrophage activation syndrome in Still's disease: analysis of clinical characteristics and survival in paediatric and adult patients. Clin Rheumatol 2017;36:2839-45.

41. Ruscitti P, Cipriani P, Di Benedetto P, Liakouli V, Carubbi F, Berardicurti $\mathrm{O}$, et al. Advances in immunopathogenesis of macrophage activation syndrome during rheumatic inflammatory diseases: toward new therapeutic targets? Expert Rev Clin Immunol 2017;13:1041-7.

42. Grom AA, Ilowite NT, Pascual V, Brunner HI, Martini A, Lovell D, et al. Rate and clinical presentation of macrophage activation syndrome in patients with systemic juvenile idiopathic arthritis treated with canakinumab. Arthritis Rheumatol 2016;68:218-28.

43. Ilowite NT, Prather K, Lokhnygina Y, Schanberg LE, Elder M, Milojevic D, et al. Randomized, double-blind, placebo-controlled trial of the efficacy and safety of rilonacept in the treatment of systemic juvenile idiopathic arthritis. Arthritis Rheumatol 2014;66:2570-9.

44. Yokota S, Itoh Y, Morio T, Sumitomo N, Daimaru K, Minota S. Macrophage activation syndrome in patients with systemic juvenile idiopathic arthritis under treatment with tocilizumab. J Rheumatol 2015;42:712-22.

45. Yokota S, Imagawa T, Mori M, Miyamae T, Takei S, Iwata N, et al. Longterm safety and effectiveness of the anti-interleukin 6 receptor monoclonal antibody tocilizumab in patients with systemic juvenile idiopathic arthritis in Japan. J Rheumatol 2014;41:759-67. 
46. Tay GC, Sauer DA, Andersen PE. Rheumatoid nodule presenting as a buccal submucosal nodule: A rare presentation of a common disease. Head Neck 2017;39:E12-4.

47. Ursini F, Naty S, Mazzei V, Spagnolo F, Grembiale RD. Kaposi's sarcoma in a psoriatic arthritis patient treated with infliximab. Int Immunopharmacol 2010;10:827-8.

48. Ursini F, Grembiale A, Naty S, Grembiale RD. Serum complement $\mathrm{C} 3$ correlates with insulin resistance in never treated psoriatic arthritis patients. Clin Rheumatol 2014;33:1759-64.

49. Iaconetta G, Friscia M, Dell'Aversana Orabona G, de Biasi S,
Romano A, Piombino P, et al. Castleman's disease mimicking a parotid gland tumor: report of a case and review of the literature. Eur Rev Med Pharmacol Sci 2014;18:1241-6.

50. Ursini F, D’Angelo S, Padula A, Gilio M, Russo E, Naty S, et al. Retrospective analysis of type 2 diabetes prevalence in a systemic sclerosis cohort from southern Italy: Comment on "Reduced incidence of type 1 diabetes and type 2 diabetes in systemic sclerosis: a nationwide cohort study" by Tseng et al., Joint Bone Spine 2016;83:307-13. Joint Bone Spine 2016;83:611-2. 